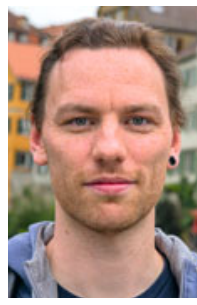

Moritz Koch

2009-2013 Bachelor Biotechnologie, FH Aachen und University of California, Berkeley, USA. 2013-2016 Master Mikrobiologie, Zusatzfach Bioethik, an der Universität Tübingen und Hebrew University Jerusalem, Israel. 2016-2020 Promotion an der Universität Tübingen und KTH Stockholm, Schweden. Seit 2020 Postdoc UBC Vancouver.

DOI: $10.1007 / \mathrm{s} 12268-021-1571-y$ (c) Der Autor 2021

Fast täglich ereilen uns Hiobsbotschaften, in welchem Bereich die Menschheit die ökologischen Belastungsgrenzen überschritten hat. Neue Rekordtemperaturen aus der Klimaforschung oder Indizien zum Artensterben: Die Notwendigkeit konsequenten Handelns ist offensichtlich. Gerade im Bereich der Lebenswissenschaften bieten sich aber auch viele Möglichkeiten, mittels technischer Innovationen Lösungen zu kreieren.

Ziel meiner Doktorarbeit war es, ein durchweg nachhaltiges Produktionssystem für Plastik zu kreieren. $\mathrm{CO}_{2}$-neutral sollte es sein, und das Produkt biologisch abbaubar, um unsere Ökosysteme nicht weiter mit Müll zu belasten. Wie geschaffen dafür schien das Cyanobakterium Synechocystis sp. PCC 6803, das mit seinem photoautrophen Stoffwechsel $\mathrm{CO}_{2}$ als Kohlenstoff- sowie Licht als Energiequelle nutzt. Zusätzlich akkumuliert Synechocystis während der Chlorose, die eine Adaption an den Stickstoffmangel darstellt, das Polymer Polyhydroxybutyrat (PHB). PHB wird schon lange als mögliche Plastikalternative im Verpackungsbereich in Betracht gezogen. Ziel unserer Arbeit war es, die intrazelluläre PHB-Produktion zu steigern, um so eine industrielle Anwendung zu ermöglichen.

In langwieriger Grundlagenforschung erweiterte unser Labor unter Karl Forchhammer das Verständnis über den PHB-Metabolismus (s. Biospektrum 02/21, S. 200) In einem ersten Projekt zeigten wir, dass das

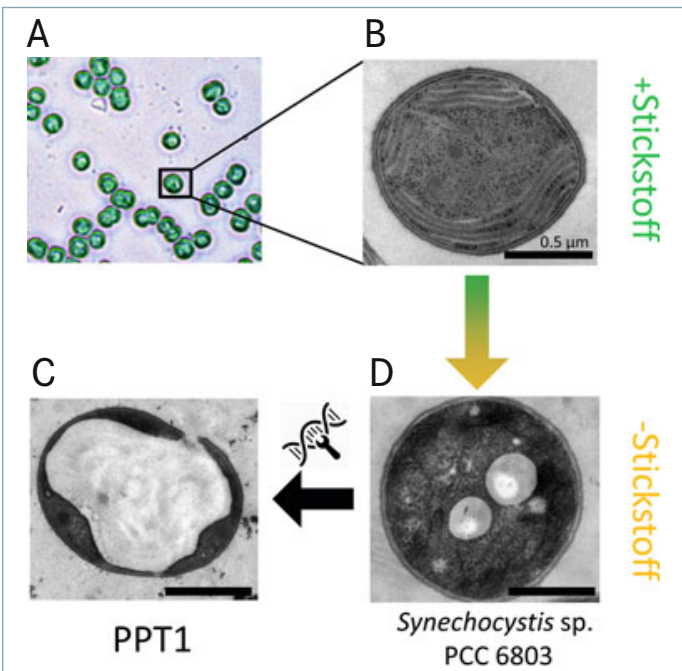

VAAM-Promotionspreis 2021

\title{
Ein nachhaltiges Produktionssystem für Plastik
}

\author{
MORITZ KOCH \\ INTERFAKULTÄRES INSTITUT FÜR MIKROBIOLOGIE UND INFEKTIONSMEDIZIN, \\ UNIVERSITÄT TÜBINGEN
}

Protein Slr0058 die Größe der PHB-Granula beeinflusst. Darüber hinaus wiesen wir einen Zusammenhang des Glykogen- und PHBStoffwechsels nach. Den Durchbruch brachte unsere Entdeckung eines zentralen Regulators, PirC, der den Kohlenstofffluss während der Chlorose steuert. Darauf aufbauend konnten wir mittels weiterer gentechnischer Veränderungen den PHB-Gehalt innerhalb der Zellen von ursprünglich zehn auf über 80 Prozent steigern [1].

Trotz dieses Erfolgs war mir klar: Es braucht deutlich mehr, um unsere Umwelt vor der Verschmutzung mit Plastikmüll zu bewahren. Technische Innovationen, wie unser Bioplastik PHB, sind lediglich ein kleines Puzzlestück in einem notwendigen Netz von Interventionen.

Wenn wir globale Probleme wie den Klimawandel und die Plastikverschmutzung in den Griff bekommen wollen, brauchen wir umfassende Änderungen. Entscheidend dafür sind gemeinsame gesellschaftliche und politische Ansätze [2]. Daher müssen wir aus der (natur)wissenschaftlichen Community uns im gesellschaftlichen Diskurs engagieren [3]. In der Corona-Pandemie haben wir gesehen, wie essenziell es ist, dass die Wissenschaft nicht nur Daten produziert, sondern auch aktiv dabei hilft, diese zu verstehen. Dies ist nach meinem Verständnis Teil unserer Verantwortung als wissenschaftliche Gemeinschaft.

Daher engagiere ich mich bei den Scientists for Future: Diese fordern eine gesellschaftliche Transformation, hin zu mehr Nachhaltigkeit, im Einklang mit naturwissenschaftlicher Empirie. Eine Kernaussage ist, dass aktuelle (politische) Maßnahmen nicht ausreichen, um die planetaren Belastungsgrenzen

Abb. 1: Licht- (A) und Elektronenmikroskopieaufnahmen des Cyanobakteriums Synechocystis sp. PCC 6803. B, Während des vegetativen Wachstums produzieren die Zellen kein PHB. C, Unter Stickstoffmangel akkumulieren sie etwa $10 \%$ PHB/Zelltrockengewicht. D, Mittels metabolic engineering konnte der PHB-Anteil auf über $80 \%$ gesteigert werden. Maßstabsbalken $=0,5 \mu \mathrm{m}$. einzuhalten. Dies trifft sowohl beim Thema Plastikverschmutzung, als auch beim Klimawandel zu.

Jede/r von uns kann aktiv werden. Damit wir in Zukunft eine Antwort auf die Frage haben „Was hast $d u$ eigentlich gegen den Klimawandel gemacht, als er noch aufzuhalten war?".

\section{Danksagung}

Die mit Abstand wichtigste Person für meine Dissertation war Karl Forchhammer. Ihm gebührt der größte Dank. Darüber hinaus der Studienstiftung des deutschen Volkes für die Finanzierung meiner Promotion, dank der ich mein eigenes Forschungsprojekt konzipieren und durchführen konnte.

\section{Literatur}

[1] Koch M, Bruckmoser J, Scholl J et al. (2020) Maximizing PHB content in Synechocystis sp. PCC 6803: a new metabolic engineering strategy based on the regulator PirC. Microb Cell Fact 19: 231

[2] Reddy S, Lau W, et al. (2020). Breaking the plastic wave: top findings for preventing plastic pollution. Report by The Pew Charitable Trusts \& SYSTEMIQ. https://www.pewtrusts. org/en/research-and-analysis/articles/2020/07/23/breakingthe-plastic-wave-top-findings

[3] Barnosky A, Ehrlich P, Hadly E (2016) Avoiding collapse: grand challenges for science and society to solve by 2050 . Element Sci Ant 4: 000094

Funding note: Open Access funding enabled and organized by Projekt DEAL. Open Access: Dieser Artikel wird unter der Creative Commons Namensnennu 4.0 International Lizenz veröffentlicht, welche die Nutzung, Vervielfältigung Bearbeitung, Verbreitung und Wiedergabe in jeglichem Medium und Forma erlaubt, sofern Sie den/die ursprünglichen Autor(en) und die Quelle ordnungsgemäß nennen, einen Link zur Creative Commons Lizenz beifügen und angeben, ob Änderungen vorgenommen wurden. Die in diesem Artikel enthaltenen Bilder und sonstiges Drittmaterial unterliegen ebenfalls der genannten Creative Commons Lizenz, sofern sich aus der Abbildungsle
nichts anderes ergibt. Sofern das betreffende Material nicht unter der genannten Creative Commons Lizenz steht und die betreffende Handlung nicht nach gesetzlichen Vorschriften erlaubt ist, ist für die oben aufgeführten Weiterverwendungen des Materials die Einwilligung des jeweiligen Rechteinhabers einzuholen. Weitere Details zur Lizenz entnehmen Sie bitte der Lizenzinformation auf http://creativecommons.org/licenses/by/4.0/deed.de.

Korrespondenzadresse:

Dr. Moritz Koch

Interfakultäres Institut für Mikrobiologie und Infektionsmedizin

Auf der Morgenstelle 28

D-72076 Tübingen

moritz.koch@uni-tuebingen.de

University of British Columbia

Life Sciences Institute

2350 Health Sciences Mall

Vancouver, BC V6T 2A1

Kanada

moritz.koch@ubc.ca

Wir danken den Sponsoren der VAAMPromotionspreise: BASF SE, New England Biolabs GmbH, Bayer Health AG, Evonik Industries AG. 